\title{
LA PESTE PORCINE AFRICAINE EN EUROPE
}

\author{
AFRICAN SWINE FEVER IN EUROPE
}

Par Marie-Frédérique LE POTIER ${ }^{1}$

(Communication présentée le 3 février 2021, manuscrit accepté le 25 avril 2021)

\begin{abstract}
RÉSUMÉ
La peste porcine africaine (PPA) est une maladie, non zoonotique, qui affecte les suidés. Elle provoque une fièvre hémorragique souvent létale chez les porcs domestiques et les sangliers européens alors qu'elle est asymptomatique chez les suidés africains adultes. Cette infection est due à un virus à ADN, double brin et enveloppé, seul membre de la famille des Asfarviridae, qui peut aussi infecter des tiques molles du genre Ornithodoros, vecteur non obligatoire. La PPA, endémique en Afrique, a été réintroduite sur le continent européen en 2007, via la Géorgie, et a depuis diffusé largement jusqu'à atteindre I'Union européenne en 2014, la Chine en 2018 avant de s'étendre à l'Asie du Sud-Est et aux îles du Pacifique. La haute résistance du virus dans l'environnement a favorisé sa large dispersion géographique en lien avec les activités humaines. Actuellement, en l'absence de vaccin ou de traitement disponible, seule l'application de mesures drastiques de biosécurité peut prévenir l'introduction en élevage.
\end{abstract}

Mots-clés : Peste Porcine Africaine, Europe, virus, vecteurs, épidémiologie, vaccins, traitements

\begin{abstract}
-ABSTRACT
African swine fever (ASF) is a non-zoonotic disease that causes a hemorrhagic fever often lethal for domestic pigs or European wild boar, while it is asymptomatic in African wild suids. ASF is due to a double strand, enveloped, DNA virus, single member of the Asfarviridae family, that can also infect soft ticks of Ornithodoros spp., a non-compulsory competent vector. ASF, historically endemic in Africa, has been re-introduced on the European continent in 2007, in Georgia, then has spread to reach the European Union in 2014, China in 2018 and further Eastern Asia and Pacific islands. ASF spread in Eurasia is essentially "man-made", in relation to the environmental resistance of the ASF Virus. As no vaccines or treatments are currently available, disease control measures rely on high level of biosecurity at herd level to prevent ASF introduction.
\end{abstract}

Key-Words: African swine fever, Europe, virus, vectors, epidemiology, vaccines, treatments

\section{INTRODUCTION}

La peste porcine africaine (PPA) est une maladie spécifique des suidés domestiques et sauvages qui n'est pas transmissible à l'homme. Elle a été décrite la première fois en 1910 au Kenya (Montgomery, 1921), mais est probablement présente depuis plusieurs centaines d'années, au sein d'un cycle sauvage entre phacochères et tiques molles du genre Ornithodoros. Elle a été introduite à diverses reprises sur les continents européen et américain depuis l'Afrique, dans les années 1960-1990, où elle a été responsable de foyers sporadiques. Elle a cependant persisté plus longuement dans la péninsule ibérique puisqu'elle n'a pu être éradiquée qu'en 1995. La présence de tiques molles de l'espèce O. erraticus, compétentes sur le plan vectoriel dans le sud de l'Espagne et du Portugal, a probablement participé aux difficultés rencontrées dans l'éradication. La prévalence de la maladie qui persiste en Sardaigne suite à son introduction en 1978, a diminué depuis la mise en œuvre du dernier plan d'éradication initié en 2015, avec l'élimination systématique des porcs errants (Franzoni et al., 2020). Le dernier foyer domestique a été déclaré en septembre 2018 et le nombre de cas chez les sangliers est en diminution, voire quasi-absent dans une des régions affectées (EFSA, 2021).

1 : Chef d'Unité Virologie Immunologie Porcine, Responsable du laboratoire national de référence PPA. Anses Ploufragan-Plouzané-Niort, site de Ploufragan. Courriel : marie-frederique.lepotier@anses.fr 
En 2007, le virus est de nouveau introduit sur le continent européen, via le débarquement de viande de porc contaminée au port de Poti, en Géorgie. Il va ensuite diffuser largement au sein de la fédération de Russie, notamment en élevage en lien avec les activités humaines, et impactera ponctuellement des populations de sangliers, suite à la consommation de viande de porcs contaminés. En 2014, il atteint la frontière Est de l'Union européenne (Pologne, Pays Baltes) où les populations de sangliers sont alors largement affectées, en plus des élevages. La maladie a depuis atteint plusieurs états membres de l'UE. Les États comme la République Tchèque et la Belgique où le virus n'a affecté que des populations de sangliers sauvages ont réussi à l'éradiquer en deux ans au prix de méthodes de lutte incluant un fractionnement du paysage (clôtures) et une diminution drastique de la densité de sangliers dans les zones considérées. Depuis 2018, la maladie a atteint la Chine puis l'ensemble de l'Asie du Sud-Est et certaines iles du Pacifique.

Aucun vaccin n'est encore disponible pour lutter contre cette infection, malgré de nombreuses recherches menées ces dix dernières années. Le virus de la PPA est un très gros virus à ADN, qui peut coexister sous sa forme moyennement virulente avec des anticorps sériques pendant plusieurs semaines. Depuis peu, la séquence complète du génome viral est disponible pour quelques-unes des souches connues, permettant ainsi d'identifier progressivement des gènes potentiellement impliqués dans la virulence. Une meilleure connaissance de la relation entre le virus et la cellule hôte est indispensable pour comprendre la manière dont le virus détourne la réponse immunitaire à son profit, et pouvoir ainsi développer des stratégies vaccinales adaptées.

\section{LE VIRUS DE LA PESTE PORCINE AFRICAINE}

Le virus de la PPA est le seul membre de la famille des Asfarviridae et du genre Asfarvirus. C'est un virus à ADN double brin, de grande taille, qui se réplique dans le cytoplasme de la cellule et ne ressemble à aucun autre virus décrit.

L'ADN génomique est protégé par une matrice, elle-même entourée d'une membrane lipidique puis d'une capside protéique. A maturité, les particules virales présentent une deuxième membrane externe, acquise lors du bourgeonnement à travers la membrane cellulaire (Figure 1). Les deux types de virions, avec ou sans membrane externe, sont infectieux (Alonso et al. 2018).
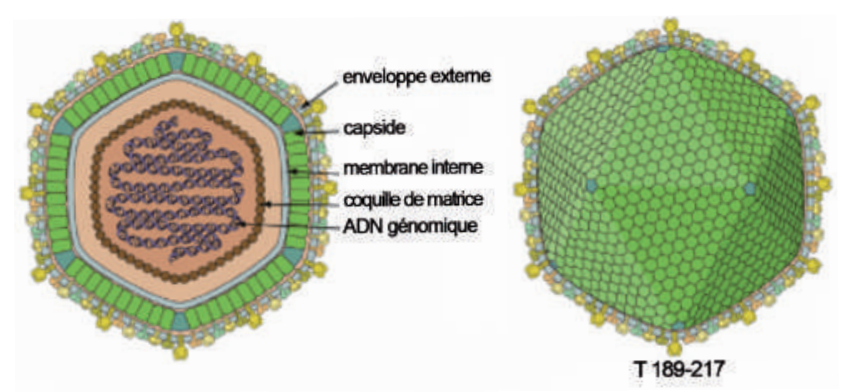

Figure 1 : Schéma de la particule virale (Source: ViralZone, Swiss Institute of Bioinformatics)
Le virus est très résistant, notamment lorsqu'il est protégé par de la matière organique (viande, sang...). Il va survivre des semaines voire des mois dans de la viande fraiche ou congelée, des produits de salaison, des cadavres de sanglier, mais aussi plusieurs jours sur du matériel (bottes, véhicules...).

La taille du génome viral varie entre 170 et $193 \mathrm{kpb}$ et code pour 150 à 167 protéines, dont celles requises pour la réplication virale. La taille du génome varie essentiellement en fonction de l'acquisition ou de la perte de gènes de la famille des multigènes (MGF) (Dixon et al. 2013), phénomène qui pourrait être dû à des recombinaisons homologues (Zhu et al. 2019).

Sur la base de la séquence partielle du gène codant pour la protéine de la capside (P72), vingt-quatre génotypes différents sont actuellement décrits en Afrique, contre seulement deux en Europe, le génotype I en Sardaigne, suite à l'introduction du virus en 1978, et le génotype II sur le continent eurasien, depuis son introduction en 2007.

Les études plus approfondies des séquences moléculaires des souches isolées depuis 2007 au travers du continent européen et plus récemment en Asie, ont confirmé qu'elles dérivaient toutes de la souche Georgia 2007/01, même si certaines présentent des évolutions sur d'autres régions du génome (CVR, IGR et MGF) définissant de nouveaux clusters, associés à une géolocalisation, confirmant ainsi l'introduction unique puis la diffusion du virus à l'échelle du continent eurasien (Gallardo et al. 2018). Actuellement, moins d'une vingtaine de souches ont pu être séquencées complètement en raison de l'extrême complexité du génome du virus de la PPA (Dixon et al. 2019).

\section{PATHOGÉNIE}

Les macrophages et les monocytes sont les principales cellules cibles du virus pour sa réplication. La manipulation des fonctions du macrophage par le virus est déterminante dans les mécanismes de pathogénie et d'évasion immunitaire (Karger et al. 2019). Plusieurs gènes ne sont pas essentiels pour la réplication virale, dont certains inhibiteurs de la réponse de l'hôte, comme les inhibiteurs de la réponse interféron de type I ou de l'apoptose (Dixon et al. 2019).

L'infection est associée à une lymphopénie et une thrombopénie, à une destruction des cellules endothéliales vasculaires et à l'induction d'une apoptose massive des lymphocytes non infectés dans le sang comme dans les organes lymphoïdes.

Des souches hautement virulentes peuvent provoquer des formes cliniques aiguës qui peuvent induire jusqu'à 100\% de mortalité chez le porc domestique ou le sanglier européen en moins de 7 jours. La mortalité est plus faible (30-70\%) pour la forme subaiguë de la maladie, due à des souches modérément virulentes. Les premiers signes cliniques des formes aiguës, perte d'appétit et léthargie, sont associés à une forte hyperthermie $\left(>41^{\circ} \mathrm{C}\right)$. Les signes cliniques apparaissent en général entre 3 à 5 jours post-inoculation en conditions expérimentales, selon la voie d'inoculation et la dose administrée, et ceci de manière comparable chez le porc et le sanglier européen. Rapidement, les animaux arrêtent de se nourrir et dépérissent. Des diarrhées hémorragiques, des vomissements, ou des avortements sont observés. Les principales lésions sont associées à des hémorragies pouvant induire un érythème dermique, de l'œedème pulmo- 
naire, une splénomégalie avec une rate déstructurée et élargie, des ganglions lymphatiques congestionnés et hémorragiques, des pétéchies sur les reins, la vessie, les poumons. Les souches faiblement virulentes vont induire une forme chronique avec essentiellement un retard de croissance, des oedèmes des articulations et des ulcères dermiques. Ces animaux peuvent survivre plusieurs semaines, voire récupérer.

L'absence d'expression clinique chez les suidés africains (phacochères et potamochères), pour lesquels la virémie n'est détectable que chez les jeunes et à un niveau maitrisé, pourrait être due à une résistance génétique dont la caractérisation ouvrirait des perspectives intéressantes pour la mise au point de vaccins, voire pour la sélection de porcs résistants (Netherton et al. 2019a). Une autre hypothèse sur l'origine de cette résistance naturelle a été argumentée récemment, suite à une étude comparative de la sensibilité de porcs et de phacochères à l'infection par le virus de la PPA, qui a démontré l'influence protectrice du microbiote intestinal (Zhang et al. 2020).

\section{HÔTES, TRANSMISSION, VECTEURS, CONTAGIOSITÉ}

\section{Hôtes et cycles de transmission}

Traditionnellement en Afrique, l'infection implique un cycle sauvage de transmission entre phacochères et tiques molles du genre Ornithodoros, sans induire de signes cliniques apparents chez le phacochère adulte, seule une virémie est décrite chez le jeune (Plowright et al. 1994). Cette persistance chronique dans la faune sauvage est à l'origine du cycle entre les tiques et le porc domestique conduisant à l'expression clinique de la maladie chez cet hôte très réceptif. Ce cycle a été décrit dans certaines parties de l'Afrique subsaharienne, mais a également joué un rôle important dans la persistance de la maladie lors de l'épizootie dans la péninsule ibérique dans les années 60 et 70 (Boinas et al. 2011). Le rôle des potamochères dans le cycle sauvage est moindre, en raison d'interactions très rares avec les tiques, et d'une prévalence très faible chez ces suidés (Penrith et al. 2019). Le cycle domestique est impliqué dans la grande majorité des foyers de peste porcine africaine dans le monde : le virus est alors transmis par les porcs domestiques ou par des produits porcins infectés aux porcs domestiques. Le profil épidémiologique de l'épizootie actuelle de peste porcine africaine en Europe définit un nouveau cycle épidémiologique impliquant les sangliers et leur environnement. Ce cycle se caractérise à la fois par une transmission directe entre sangliers et par une transmission indirecte via l'environnement où la contamination persiste via les cadavres, la persistance du virus dans l'environnement étant favorisée par le froid et l'humidité et le climat du pays (Chenais et al. 2019).

Les modalités de transmission incluent la voie directe (de porc/sanglier infecté à porc/sanglier sensible) et la voie indirecte compte-tenu de l'importante résistance du virus (homme, seringues, matériels, véhicules souillés, produits d'origine animale, aliments contenant des produits porcins dont déchets de cuisine et denrées alimentaires). Les animaux infectés excrètent le virus dans la plupart des fluides et excrétas corporels incluant le sang, fluide nasal, fèces et urine (Guinat et al. 2014), mais la charge virale est très largement supérieure dans le sang, ce qui implique une transmission essentiellement par contact très proche entre animaux plutôt que par voie aérienne.

\section{Vecteurs}

La question du rôle que pourraient jouer des vecteurs potentiels comme les tiques molles en Europe a été régulièrement posée depuis 2014. Afin de répondre à cette question, des études ont été menées en collaboration entre l'Anses et le CIRAD sur la compétence vectorielle vis-à-vis de la souche virale circulante en Eurasie (Georgia 2007/1), des espèces d'Ornithodoros présentes en Europe (O. erraticus et O. verrucosus), en comparaison avec la tique africaine O. moubata. Dans nos conditions expérimentales, ni O. erraticus, ni O. verrucosus infectées n'ont réussi à transmettre la souche virale Georgia 2007/1 à des porcs sains, contrairement à O. moubata. (Pereira de Oliveira et al. 2019). Cependant, les deux espèces européennes maintiennent le virus infectieux plusieurs semaines (Pereira de Oliveira et al. 2020a). De plus, lorsque que l'on donne à manger des tiques O. erraticus infectées à un porc, il déclenche la maladie (Pereira de Oliveira et al. 2020b). Ces tiques pourraient donc potentiellement jouer un rôle de réservoir. Les tiques molles étant endophiles et nidicoles, on ne les retrouve que dans certaines zones géographiques en Europe où les conditions climatiques (température et hygrométrie) leur sont favorables, actuellement principalement au sud du Portugal et de l'Espagne et près de la mer Noire (ECDC \& EFSA, 2020), mais leur habitat est en constante régression. Sur le territoire métropolitain, le risque de transmission et de réservoir associé a en conséquence était considéré comme quasi-nul (Saegerman et al. 2020).

L'existence de vecteurs mécaniques représentés par des arthropodes piqueurs est régulièrement évoquée dans les Pays de l'Est de l'UE notamment en raison d'une plus forte pression infectieuse en élevage pendant les mois d'été. Leur implication dans la diffusion de l'infection reposerait sur leurs capacités à agir en tant que vecteurs mécaniques. Les hématophages tels que les stomoxes ou les tabanidés pourraient en théorie transmettre du sang infecté à un autre hôte lors de repas de sang interrompus (Bonnet et al. 2020 ; Vergne et al. 2020) mais la preuve de leur implication doit être encore démontrée par des données expérimentales contrôlées ainsi que des données sur leur fréquence en élevage de porcs ou en forêt.

\section{Contagiosité}

La PPA a été longtemps décrite comme une maladie hautement contagieuse, mais les données acquises depuis sa réintroduction au sein de l'UE, aussi bien sur le terrain que lors d'expérimentations contrôlées ont remis en cause cette définition. La PPA diffuse lentement en élevage, de porc à porc majoritairement suite à des contacts avec des épanchements sanguins. En général, après l'introduction dans un élevage, la mortalité est très faible les deux premières semaines, puis augmente progressivement jusqu'à atteindre un fort taux. Chez les sangliers sauvages, la propagation de la maladie se fait de même de 
proche en proche. Les sauts de plusieurs milliers de kilomètres qui ont pu être observés sont à relier à l'activité humaine, le virus pouvant résister très longtemps dans la viande ou sur des matériels souillés.

\section{ÉPIDÉMIOLOGIE EN EUROPE}

Depuis sa réintroduction sur le continent européen en avril 2007, la PPA a diffusé au travers du Caucase et de la fédération de Russie où elle s'est installée au sein des élevages de porcs et des populations de sangliers sauvages (Gogin et al. 2013).

En 2014, la PPA a atteint les états de l'est de l'Union Européenne, la Pologne et les Pays Baltes affectant là aussi porcs et sangliers, puis a diffusé à l'occasion d'erreurs humaines en Moldavie (2016), Roumanie (2017), République Tchèque (2017), Hongrie (avril 2018), Belgique (septembre 2018), Slovaquie (juillet 2019), Serbie (août 2019), et Grèce (février 2020), ou de proche en proche au sein de populations de sangliers comme à la frontière entre la Pologne et l'Allemagne (septembre 2020). Au sein de l'Union européenne (UE), la plupart des élevages de porcs affectés étaient de petits élevages familiaux, mais quelques élevages industriels avec un niveau de biosécurité bien supérieur ont aussi été atteints. Ces foyers domestiques ont été le plus souvent éradiqués rapidement, à l'exception de la Roumanie, où la diffusion est difficile à maitriser. En revanche, la maladie s'est installée dans les populations de sangliers sauvages dans plusieurs régions de l'UE, notamment dans les régions très forestières comme en Pologne ou dans les États baltes. La situation épidémiologique évoluant rapidement, un bulletin hebdomadaire est publié et consultable sur le site de la plateforme d'épidémiosurveillance en santé animale : https://www.plateforme-esa.fr/.

\section{MÉTHODES DE LUTTE ACTUELLES}

En l'absence de vaccin ou de traitement, la prévention de l'introduction en élevage repose sur des mesures de biosécurité drastiques afin de préserver les animaux de contacts à risque. Les méthodes de lutte au sein de l'UE consistent en une détection rapide de la maladie, à l'abattage du foyer infecté et à un blocage des mouvements des élevages alentours. La présence d'un foyer infecté va induire la mise en place par l'UE de différentes zones " tampons " qui, pour éviter la propagation de la maladie, vont être soumises à des restrictions de mouvements vers les zones indemnes (Décision EU 2014/709).

Chez les sangliers sauvages, la stratégie consistant à suspendre la chasse au cour du foyer ainsi que toute activité forestière pouvant déranger les sangliers, à fractionner le paysage par des clôtures, et à réduire drastiquement le nombre de sangliers présents dans la zone limitrophe du foyer, appelée "zone blanche " a permis à la République Tchèque comme à la Belgique de retrouver en deux ans leur statut indemne. Cependant, cette stratégie pourrait s'avérer plus délicate à appliquer lorsque le foyer d'infection se trouve sur une zone plus difficile à délimiter en raison de sa géographie et de son paysage (montagnes, zones humides...).

\section{PERSPECTIVES D'UNE VACCINATION CONTRE LE VIRUS DE LA PESTE PORCINE AFRICAINE}

Différentes stratégies de développement de vaccins ont été tentées avec des succès divers. L'administration de virus inactivé en absence ou en présence d'adjuvants modernes n'a induit aucune protection, alors que les candidats vaccins avaient induit une séroconversion (Blome et al. 2014). Les approches de type vaccin sous-unitaire, basé sur des protéines recombinantes ou des ADN plasmidiques n'ont pas ou peu induit de protection. Une protection partielle avec un retard de l'expression clinique a pu être obtenue par inoculation d'ADN plasmidiques, en l'absence de séroconversion (Argilaguet et al. 2012). Une protection plus complète avec une nette réduction des signes cliniques a été obtenue suite à l'immunisation avec une librairie d'ADN chez une proportion des porcs immunisés mais pas pour l'ensemble des animaux (Lacasta et al. 2014). La plupart des approches vectorielles développées n'ont pas non plus permis de protéger les porcs, et ce quel que soit le vecteur utilisé (Jankovich et al. 2018 ; Netherton et al. 2019b). Récemment, en ayant recours à un protocole de pré-immunisation avec un cocktail de huit adénovirus recombinants suivie d'un rappel avec un vecteur recombinant du virus de la vaccine modifié "Ankara ", codant pour les mêmes huit gènes, Goatley et al. (2020) ont réussi à protéger l'ensemble des porcs vis à vis d'une épreuve avec la souche virale hyper-virulente OURT88/1 du génotype I, ces résultats ouvrent la voie pour une approche vaccinale vectorisée qui permettraient de disposer d'un vaccin candidat contre la souche du génotype II circulant actuellement en Eurasie.

Pour autant actuellement, ce sont encore les souches vivantes atténuées qui induisent la meilleure protection, notamment les souches naturellement atténuées du génotype I qui permettent une protection homologue ou croisée vis-à-vis de certains autres génotypes (King et al. 2011). Cependant, ce type de souche atténuée induit quelques inflammations et œedèmes au niveau articulaire qui ont un impact négatif sur la croissance des porcs. Des essais d'atténuation plus importante par délétion génétique de ces souches naturellement atténuées a pu conduire selon les cas, à une diminution du pouvoir protecteur (Abrams et al. 2013), comme à une meilleure protection (Reis et al. 2017). Les approches les plus récentes consistent à déléter plusieurs gènes de la région des $\mathrm{MGF}$, gènes qui inhibent la réponse Interféron de type I, pouvant ainsi atténuer la virulence de la souche modifiée chez le porc et le protéger contre une épreuve par le virus parental (O'Donnell et al. 2015). Par ailleurs, Borca et al. (2020) ont pu protéger des porcs en les vaccinant avec une souche Georgia délétée pour le gène I177L, vis-à-vis de la souche parentale inoculée 28 jours plus tard. La souche délétée induisait une virémie sur une période de 28 jours mais n'était pas transmise aux porcs sentinelles. Cependant, cette souche délétée se cultive moins bien sur macrophages que la souche parentale. Ces quelques exemples illustrent la difficulté de développer un vaccin contre la PPA selon les méthodes éprouvées pour d'autres maladies. Les principales limites sont aujourd'hui liées à la difficulté de produire un virus vivant suffisamment atténué pour ne pas diffuser ou rendre malades les animaux tout en conservant un potentiel protecteur vis-à-vis 
du virus sauvage. Le vaccin doit pouvoir aussi induire une réponse sérologique distinguable d'une infection par le virus sauvage et être suffisamment stable pour être délivré dans l'environnement à destination des sangliers sauvages. Le déficit de connaissance du génome du virus est en train de se combler, grâce aux nouvelles méthodes de séquençage profond. Pour autant, les fonctions de plus de 150 protéines virales sont loin d'être identifiées, et tous les gènes de virulence ne sont pas encore connus. Ce virus interfère avec les réponses innées, et les corollaires de protection ne sont pas encore identifiés. De plus, les virus vivants atténués, actuellement brevetés, se cultivent sur cellules primaires (macrophages), ce qui est incompatible avec une production industrielle (Arias et al. 2017).

\section{AUTRES TRAITEMENTS}

Quelques rares études publiées évoquent des pistes de traitement. Le virus de la PPA est le seul virus connu comme étant capable de coder sa propre Topoisomérase II. L'action de certains antibiotiques (Fluoroquinolones, Isoflavone...) sur cette enzyme entraine une inhibition du cycle de réplication in vitro (Freitas et al. 2016 ; Coelho \& Leitão, 2020).

Par ailleurs, Hübner et al. (2018) ont montré, en utilisant la technologie CRIPS/Cas9, que la suppression du gène de la P30 inhibe la réplication du virus de la PPA, in vitro. Cette nouvelle approche pourrait permettre d'identifier des pistes pour le développement d'antiviraux spécifiques, voire d'imaginer l'induction d'une résistance chez des porcs transgéniques.

\section{CONCLUSION}

Longtemps peu considérée par les principaux pays producteurs porcins (Europe, Asie, Amérique), la peste porcine africaine est devenue, en quelques années, l'enjeu majeur de cette filière. Même si de nombreuses équipes de recherche se sont investies dans la mise au point de candidats vaccins, l'obtention d'un vaccin efficace et sécurisé semble difficilement atteignable à très court terme. La pression d'infection représentée aujourd'hui par de multiples foyers sauvages ou domestiques non maîtrisés en Europe comme en Asie suggère un risque considérable d'introduction de la maladie à tout instant sur le territoire français et dans une zone qu'il est difficile de prévoir compte-tenu de son caractère aléatoire, à relier à des erreurs humaines. Cependant, des mesures de biosécurité bien appliquées suffisent à protéger un élevage, en évitant ainsi des contacts à risque. La vigilance de tous les acteurs et la mise en place de dispositifs de surveillance efficaces se révèlent incontournables alors que l'épizootie continue de progresser en Europe comme en Asie.

\section{BIBLIOGRAPHIE}

- Abrams CC, Goatley L, Fishbourne E, Chapman D, Cooke L, Oura CA et al. Deletion of virulence associated genes from attenuated African swine fever virus isolate OUR T88/3 decreases its ability to protect against challenge with virulent virus. Virology. 2013; 443, 99-105.

- Alonso C, Borca M, Dixon L, Revilla Y, Rodriguez F, Escribano JM. ICTV Report Consortium. ICTV Virus Taxonomy Profile: Asfarviridae. J. Gen. Virol. 2018; 99, 613-614.

- Argilaguet JM, Perez-Martin E, Nofrarias M, Gallardo C, Accensi F, Lacasta $A$ et al. DNA vaccination partially protects against African swine fever virus lethal challenge in the absence of antibodies. PLoS One. 2012; 7, e40942.

- Arias M, de la Torre A, Dixon LK, Gallardo C, Jori F, Laddomada A et al. Approaches and perspectives for development of African swine fever virus vaccines. Vaccines (Basel). 2017 ; 5, 35.

- Blome S, Gabriel C, Beer M. Modern adjuvants do not enhance the efficacy of an inactivated African swine fever virus vaccine preparation. Vaccine. 2014; 32, 3879-3882.

- Boinas FS, Wilson AJ, Hutchings GH,
Martins C, Dixon L. The persistence of African swine fever virus in field-infected Ornithodoros erraticus during the ASF endemic period in Portugal. PloS one. 2011; 6, e20383.

- Bonnet S, Bouhsira E, De Regge N, Fite J, Etore F, Garigliany M-M et al. Putative role of arthropod vectors in African swine fever virus transmission in relation to their bio-ecological properties. Viruses. 2020; 12(7):778.

- Borca MV, Ramirez-Medina E, Silva E, Vuono E, Rai A, Pruitt S et al. Development of a highly effective African swine fever virus vaccine by deletion of the $1177 \mathrm{~L}$ gene results in sterile immunity against the current epidemic Eurasia strain. J Virol. 2020; 94(7).

- Chenais E, Depner K, Guberti V, Dietze K, Viltrop A, Ståhl K. Epidemiological considerations on African swine fever in Europe 2014-2018. Porcine Health Management. 2019 ; 5, 6.

- Coelho J \& Leitão A. The African swine fever virus (ASFV) Topoisomerase II as a target for viral prevention and control. Vaccines (Basel). 2020; 8(2).

- European Commission (EC). Décision d'exécution de la Commission 2014/709/UE du 9 octobre 2014 concernant des mesures zoosanitaires de lutte contre la peste porcine africaine dans certains États membres et abrogeant la décision d'exécution 2014/178/UE. 2014 ; Disponible à: http://data.europa.eu/eli/decimpl/ 2014/709/oj, (consulté le 01er février 2021).

- Dixon LK, Chapman DA, Netherton CL, Upton C. African swine fever virus replication and genomics. Virus Res. 2013; 173, 3-14.

- Dixon LK, Islam M, Nash R, Reis AL. African swine fever virus evasion of host defences. Virus Res. 2019; 266, 25-33.

- ECDC \& EFSA, European Centre for Disease Prevention and Control and European Food Safety Authority. Tick maps. Stockholm: ECDC. 2020 ; Disponible à: https://www.ecdc.eur opa.eu/en/publications-data/ornith odorus-spp-current-known-distrib ution-may-2020 (consulté le 1er février 2021).

- European Food Safety Authority (EFSA). ASF Exit Strategy: Providing cumulative evidence of the absence of African swine fever virus circulation in wild boar populations using standard 
surveillance measures. EFSA Journal 2021: 19(3):6419, 72 pp.

- Franzoni G, Dei Giudici S, Loi F, Sanna D, Floris M, Fiori $\mathrm{M}$ et al. African swine fever circulation among free-ranging pigs in Sardinia: Data from the Eradication Program. Vaccines (Basel) 2020; 8(3): 549.

- Freitas FB, Frouco G, Martins C, Leitão A, Ferreira F. In vitro inhibition of African swine fever virus-topoisomerase II disrupts viral replication. Antiviral Res. 2016; 134, 34-41.

- Gallardo C, Nurmoja I, Soler A, Delicado V, Simón A, Martin E et al. Evolution in Europe of African swine fever genotype II viruses from highly to moderately virulent. Vet. Microbiol. 2018; 219, 70-79.

- Goatley LC, Reis AL, Portugal R, Goldswain H, Shimmon GL, Hargreaves $\mathrm{Z}$ et al. A Pool of eight virally vectored African swine fever antigens protect pigs against fatal disease. Vaccines (Basel). 2020; 8(2).

- Gogin A, Gerasimov V, Malogolovkin A, Kolbasov D. African swine fever in the North Caucasus region and the Russian Federation in years 2007-2012. Virus Res. 2013; 173, 198-203.

- Guinat C, Gubbins S, Vergne T, Gonzales JL, Dixon L, Pfeiffer DU. Experimental pig-to-pig transmission dynamics for African swine fever virus, Georgia 2007/1 strain. Epidemiol. Infect. 2014; 144, 25-34.

- Hübner A, Petersen B, Keil GM, Niemann H, Mettenleiter TC, Fuchs W: Efficient inhibition of African swine fever virus replication by CRISPR/Cas9 targeting of the viral p30 gene (CP204L). Sci Rep. 2018; 8(1):1449.

- Jankovich JK, Chapman D, Hansen DT, Robida MD, Loskutov A, Craciunescu F et al. Immunisation of pigs by DNA prime and recombinant vaccinia virus boost to identify and rank African swine fever virus immunogenic and protective proteins. J. Virol. 2018; 92, e02219-02217.

- Karger A, Pérez-Núñez D, Urquiza J, Hinojar P, Alonso C, Freitas FB et al. An update on African swine fever virology. Viruses. 2019; 11, 864.

- King K, Chapman D, Argilaguet JM,
Fishbourne E, Hutet E, Cariolet R et al. Protection of European domestic pigs from virulent African isolates of African swine fever virus by experimental immunisation. Vaccine. 2011; 29, 4593-4600.

- Lacasta A, Ballester M, Monteagudo PL, Rodriguez JM, Salas ML, Accensi F et al. Expression library immunization can confer protection against African swine fever virus lethal challenge. J. Virol. 2014; 88(22):13322-32.

- Montgomery RE. On A Form of Swine Fever Occurring in British East Africa (Kenya Colony). J. Comp. Path. Therap. 1921; 34, 159-191.

- Netherton CL, Connell S, Benfield CTO, Dixon LK. The genetics of life and death: virus-host interactions underpinning resistance to African swine fever, a viral hemorrhagic disease. Front. Genet. 2019a; 10:402.

- Netherton CL, Goatley LC, Reis AL, Portugal R, Nash RH, Morgan SB et al. Identification and immunogenicity of African swine fever virus antigens. Front. Immunol. 2019b; 10:1318.

- O'Donnell V, Holinka LG, Krug PW, Gladue DP, Carlson J, Sanford B et al. African swine fever virus Georgia 2007 with a deletion of virulence-associated gene 9GL (B119L), when administered at low doses, leads to virus attenuation in swine and induces an effective protection against homologous challenge. J. Virol. 2015; 89, 8556-8566.

- Penrith M-L, Bastos AD, Etter EMC, Beltrán-Alcrudo D. Epidemiology of African swine fever in Africa today: sylvatic cycle versus socio-economic imperatives. Transbound Emerg Dis. 2019; 66: 672- 686.

- Pereira de Oliveira R, Hutet E, Paboeuf F, Duhayon M, Boinas F, Perez de Leon A et al. Comparative vector competence of the Afrotropical soft tick Ornithodoros moubata and Palearctic species, $\mathrm{O}$. erraticus and $\mathrm{O}$. verrucosus, for African swine fever virus strains circulating in Eurasia. Plos One. 2019; 14(11).

- Pereira de Oliveira R, Hutet E, Lancelot R, Paboeuf F, Duhayon M, Boinas F et al. Differential vector competence of Ornitho- doros soft ticks for African swine fever virus: What if it involves more than just crossing organic barriers in ticks? Parasites \& Vectors. 2020a; 13:618.

- Pereira de Oliveira R, Hutet E, Duhayon M, Guionnet J-M, Paboeuf F, Vial L et al. Successful infection of domestic pigs by ingestion of the European soft tick O. Erraticus that fed on African swine fever virus infected pig. Viruses. 2020b; 12(3):300.

- Plowright W, Thomson GR, Neser, JA. African swine fever. In JAW Coetzer, GR Thomson \& RC Tustin (eds.). Infectious diseases of livestock with special reference to southern Africa, Oxford University Press, Cape Town. 1994; vol. 1, pp. 568-599.

- Reis AL, Goatley LC, Jabbar T, Sanchez-Cordon PJ, Netherton CL, Chapman DAG et al. Deletion of the African swine fever virus gene DP148R does not reduce virus replication in culture but reduces virus virulence in pigs and induces high levels of protection against challenge. J. Virol. 2017; 91.

- Saegerman C, Bonnet S, Bouhsira E, De Regge N, Fite J, Etoré F et al. An expert opinion assessment of blood-feeding arthropods based on their capacity to transmit African Swine Fever virus in Metropolitan France. Transbound. Emerg. Dis. 2020; 00, 1-15.

- Vergne T, Andraud M, Bonnet S, De Regge N, Desquesnes M, Fite J et al. Mechanical transmission of African swine fever virus by Stomoxys calcitrans: Insights from a mechanistic model. Transbound Emerg. Dis. 2020; 00, 1-9.

- Zhang J, Rodríguez F, Navas MJ, Costa-Hurtado M, Almagro V, BoschCamós L et al. Fecal microbiota transplantation from warthog to pig confirms the influence of the gut microbiota on African swine fever susceptibility. Sci Rep. 2020; 10, 17605.

- Zhu Z, Xiao CT, Fan Y, Cai Z, Lu C, Zhang $\mathrm{G}$ et al. Homologous recombination shapes the genetic diversity of African swine fever viruses. Vet. Microbiol. 2019 ; 236, 108380. 